\title{
GENERALISED VANDERMONDE DETERMINANTS AND SCHUR FUNCTIONS
}

\author{
R. C. KING
}

ABSTRACT. A formula for determinants similar to that of Vandermonde is given in terms of the Vandermonde determinant and Schur functions. The Schur functions are then expressed in terms of symmetric monomial functions.

In a recent paper [1] Fink derived a formula for certain determinants arising in a differential equations problem but remarked that there perhaps ought to be a better general representation for the symmetric function appearing in this formula. This is indeed the case and the relevant symmetric function is just one of a special class of the $S$-functions introduced by Schur [2]. Moreover $S$-functions are the symmetric functions appearing in formulae for determinants which are a generalisation of those considered by Fink.

For every partition $(\lambda)=\left(\lambda_{1}, \lambda_{2}, \cdots, \lambda_{a}\right)$ of $l$ into $a$ parts, with $a \leq n$, Littlewood [3, p. 87] has proved that if the element in the $i$ th row and $j$ th column of an $n \times n$ matrix is $x_{i}^{\lambda_{j}{ }^{+} n-j}$, with $\lambda_{j}=0$ for $j>a$, then the determinant of this matrix is given by

$$
\left|x_{i}^{\lambda} i^{+n-j}\right|=\left|x_{i}^{n-j}\right|\{\lambda ; \mathbf{x}\}
$$

where $\{\lambda ; \mathbf{x}\}$ is the $S$-function in the $n$ variables $\left(x_{1}, x_{2}, \ldots, x_{n}\right)$ specified by the partition $(\lambda)$. The determinant $\left|x_{i}^{n-j}\right|$ is essentially the same as the Vandermonde determinant, $V_{n}(\mathbf{x})$, since

$$
\left|x_{i}^{n-j}\right|=\prod_{i<j}\left(x_{i}-x_{j}\right)=(-)^{n(n-1) / 2} V_{n}(\mathbf{x}) .
$$

The $S$-function $\{\lambda ; \mathbf{x}\}$ is a symmetric function of the various $x_{i}$ and can itself be written in the form of the determinant of an $n \times n$ matrix whose elements are homogeneous product sums denoted by $h_{r}(\mathbf{x})$. Each such symmetric function $h_{r}(\mathbf{x})$, with $r$ a positive integer, is the sum of all the monomial symmetric functions $P(\rho ; \mathbf{x})$ of degree $r$ in the various $x_{i}$, that is

$$
h_{r}(\mathbf{x})=\sum_{\rho} P(\rho ; \mathbf{x}) \quad \text { if } r>0,
$$

Received by the editors October 26, 1973.

AMS (MOS) subject classifications (1970). Primary $15 A 15$.

Key words and phrases. Vandermonde determinant, Schur functions.

Copyright () 1975, American Mathematical Society 
where the summation is carried out over all partitions $(\rho)=\left(\rho_{1}, \rho_{2}, \cdots, \rho_{b}\right)$ of $r$ into $b$ parts, and

$$
P(\rho ; \mathbf{x})=\sum_{(i)} x_{i_{1}}^{\rho_{1}} x_{i_{2}}^{\rho_{2}} \cdots x_{i_{b}}^{\rho_{b}}
$$

with this sum taken over all possible sets $\left(i_{1}, i_{2}, \ldots, i_{b}\right)$ of $b$ distinct numbers chosen from the set $(1,2, \cdots, n)$.

It is convenient also to define $h_{r}(\mathbf{x})=0$ for $r<0$ and $h_{r}(\mathbf{x})=1$ for $r=0$. With this notation $[3$, p. 88], [4, p. 118], [5, p. 59]:

$$
\{\lambda ; \mathbf{x}\}=\left|h_{\lambda_{i}-i+j}(\mathbf{x})\right|,
$$

and this determinant may be expanded to give

$$
\{\lambda ; \mathbf{x}\}=\sum_{\mu} b_{\lambda^{\prime h} \mu_{1}}^{\mu_{1}}(\mathbf{x}) h_{\mu_{2}}(\mathbf{x}) \cdots h_{\mu_{c}}(\mathbf{x}),
$$

where $(\mu)=\left(\mu_{1}, \mu_{2}, \cdots, \mu_{c}\right)$ is a partition of $m$ into $c$ parts with $m=l$. The determinants $V(\alpha, n, m)$, considered by Fink, are given by

$$
V(\alpha, n, m)=(-)^{n(n-1) / 2}\left|x_{i}^{\lambda_{j}^{+n-j}}\right|
$$

with :

$$
\lambda_{i}= \begin{cases}(m-1-\alpha) & \text { if } i=1,2, \cdots, n-1-\alpha, \\ 0 & \text { if } i=n-\alpha, n-\alpha+1, \cdots, n .\end{cases}
$$

Comparison of Fink's definition

$$
V(\alpha, n, m)(\mathbf{x})=V_{n}(\mathbf{x}) g(\alpha, n, m)(\mathbf{x})
$$

with (1), (2) and (7) then yields

$$
g(\alpha, n, m)(\mathbf{x})=\left\{(m-1-\alpha)^{n-1-\alpha} ; \mathbf{x}\right\} .
$$

The formula obtained by Fink for this symmetric function $g(\alpha, n, m)(\mathbf{x})$ is just a special case of (5) which gives

$$
g(a, n, m)(\mathbf{x})=\left|h_{m-1-a-i+j}(\mathbf{x})\right| .
$$

Thus, for example,

$$
g(n-3, n, n)(\mathbf{x})=\left|\begin{array}{ll}
h_{2}(\mathbf{x}) & h_{3}(\mathbf{x}) \\
h_{1}(\mathbf{x}) & h_{2}(\mathbf{x})
\end{array}\right| .
$$

It was pointed out by Fink that this may be reduced to

$$
g(n-3, n, n)(\mathbf{x})=P\left(2^{2} ; \mathbf{x}\right)+P\left(21^{2} ; \mathbf{x}\right)+2 P\left(1^{4} ; \mathbf{x}\right)
$$

The generalisation of this simplified result follows immediately from the 
well-known identity [4, p. 164], [5, p. 59]

$$
\{\lambda ; \mathbf{x}\}=\sum_{\rho} m_{\rho}^{\lambda} P(\rho ; \mathbf{x}),
$$

where the coefficients $m_{\lambda}^{\mu}$ are defined by the product

$$
h_{\lambda_{1}}(\mathbf{x}) h_{\lambda_{2}}(\mathbf{x}) \cdots h_{\lambda_{a}}(\mathbf{x})=\sum_{\mu} m_{\lambda}^{\mu}\{\mu ; \mathbf{x}\} .
$$

Thus the general result corresponding to the determinants considered by Fink is

$$
g(\alpha, n, m)(\mathbf{x})=\sum_{\mu} m_{\mu}^{(m-1-\alpha)^{n-1-\alpha}} P(\mu ; \mathbf{x}) .
$$

Comparison of (6) and (14) shows that the coefficients $b_{\lambda}^{\mu}$ and $m_{\lambda}^{\mu}$ may be considered to be elements of matrices $B$ and $M$ whose rows and columns are labelled by partitions $(\lambda)$ and $(\mu)$ and which are mutually inverse [6], [7]. The elements of $B$ may easily be found [3, p. 90], [4, p. 163], [6], [7], [8] by expanding the determinant of (5). It is clear that if the ordering of partitions used to label rows and columns is lexicographical, then $B$ is triangular with diagonal elements all unity. The inversion of $B$ to give $M$ is then straightforward.

Alternatively (14) may be used directly to obtain $M$ by noting that (5) implies

$$
h_{r}(\mathbf{x})=\{r ; \mathbf{x}\}
$$

and by making use of the Littlewood-Richardson [3, p. 94], [5, p. 61], [8] rule for the evaluation of products of $S$-functions. Only a simplified version of this rule is required here since the $S$-function (16) corresponds to a partition $(r)$ into one part only. This rule is then

$$
\{\sigma ; \mathbf{x}\}\{r ; \mathbf{x}\}=\sum_{\tau}\{r ; \mathbf{x}\},
$$

where $(\sigma)$ and $(r)$ are partitions of $s$ and $t$ with $t=s+r$, and the summation is carried out over all $(r)$ such that $\tau_{1} \geq \sigma_{1} \geq \tau_{2} \geq \sigma_{2} \cdots$.

It is worth pointing out that the result

$$
\left|x_{i}^{\lambda_{j}^{+n-j}}\right|=\left|x_{i}^{n-j}\right|\left|h_{\lambda_{i}-i+j}(\mathbf{x})\right|
$$

has been attributed by Littlewood, Richards on and Muir [9] to Jacobi [9, vol. (i), p. 341] and Trudi [9, vol. (iii), p. 135], and that the elements of the matrix $B$ were first tabulated by Kostka [9, vol. (iv), p. 145], enabling the formula

$$
\left|x_{i}^{\lambda_{j}+n-j}\right|=\left|x_{i}^{n-j}\right| \sum_{\mu} b^{\mu} h_{\mu_{1}}(\mathbf{x}) h_{\mu_{2}}(\mathbf{x}) \cdots h_{\mu_{c}}(\mathbf{x})
$$


to be used. Of course this expression involves, in general, both positive and negative coefficients $b_{\lambda}^{\mu}$. On the other hand the final result

$$
\left|x_{i}^{\lambda_{j}+n-j}\right|=\left|x_{i}^{n-j}\right| \sum_{\mu} m_{\mu}^{\lambda} P(\mu ; \mathbf{x})
$$

involves only positive coefficients $m_{\mu}^{\lambda}$ and it would appear that this result has not previously been given explicitly, although, as has been stated, the expansion (13) is well known.

\section{REFERENCES}

1. A. M. Fink, Certain determinants related to the Vandermonde, Proc. Amer. Math. Soc. 38 (1973), 483-488. MR $47 \# 239$.

2. I. Schur, Über eine Klasse von Matrizen die sich einer gegebenen Matrix zuordnen lassen, In augural Dissertation, Berlin, 1901.

3. D. E. Littlewood, The theory of group characters, 2nd ed., University Press, Oxford, 1950.

4. F. D. Murnaghan, The theory of group representation, Johns Hopkins Press, Baltimore, Md., 1938.

5. G. de B. Robin son, Representation theory of the symmetric group, Math. Expositions, no. 12, Univ. of Toronto Press, Toronto, 1961. MR 23 \#A3182.

6. S. Blaha, Character analysis of $U(N)$ and $S U(N)$, J. Mathematical Phys. 10 (1969), 2156-2168. MR 40 \#354.

7. R. C. King and S. P. O. Plunkett, The evaluation of branching rules for linear groups using mappings between weight spaces, J. Phys. 33 (1972), 145-153.

8. D. E. Littlewood and A. R. Richardson, Group characters and algebra, Philos. Trans. Roy. Soc. London A233 (1934), 99-141.

9. T. Muir, Theory of determinants, Vols. (i), (ii), (iii) and (iv), Macmillan, London, 1906.

\section{ENGLAND}

DEPARTMENT OF MATHEMATICS, THE UNIVERSITY, SOUTHAMPTON, HANTS, 